\title{
Fractal signal processing method of acoustic emission monitoring for seismic damage of concrete columns
}

\section{Yong Huang* and Changsong Shao}

Key Lab of Structures Dynamic Behavior and Control of the Ministry of Education,

School of Civil Engineering,

Harbin Institute of Technology,

Harbin, 150090, China

and

Key Lab of Smart Prevention and Mitigation of Civil Engineering

Disasters of the Ministry of Industry and Information Technology,

Harbin Institute of Technology,

Harbin, 150090, China

Email: huangyong@hit.edu.cn

Email: shaochangsong_hit@163.com

*Corresponding author

\author{
Xin Yan \\ China Highway Hi-tech Traffic Inspection and \\ Certification Co., Ltd., \\ Beijing 100088, China \\ Email: yanxin66hrb@163.com
}

\begin{abstract}
In this paper, a new fractal theory-based acoustic emission (AE) signal processing method is proposed. It is found that both the curve lengths and fractal dimensions (FDs) of AE signal are related with damage evolution. The AE tests of pseudo-static experiment of a reinforced concrete column (RCC), a reinforced nano-concrete column and a concrete-filled glass fibre reinforced polymer (GFRP) tube are then performed for validation. For each specimen, several piezoelectric ceramic (PZT) patches and one AE sensor are bonded at different positions of the specimen surface to monitor the AE signals. The results show that the fractal theory-based damage method can assess damage evolution effectively. In addition, the damage can be localised approximately by the diversity of damage assessing index values from various PZT detectors.
\end{abstract}

Keywords: fractal dimension; acoustic emission; pseudo-static experiment; damage assessment; damage localisation.

Reference to this paper should be made as follows: Huang, Y., Shao, C. and Yan, X. (2019) 'Fractal signal processing method of acoustic emission monitoring for seismic damage of concrete columns', Int. J. Lifecycle Performance Engineering, Vol. 3, No. 1, pp.59-76. 
Biographical notes: Yong Huang received his $\mathrm{MS}$ and $\mathrm{PhD}$ degrees from the Harbin Institute of Technology in 2008 and 2012, respectively. He is currently a Professor in School of Civil Engineering, Harbin Institute of Technology, China. His research interests include structural health monitoring, system identification, Bayesian inference, and statistical and signal processing.

Changsong Shao received his BS in Civil Engineering, Qingdao University of Technology, China. He currently works as a Master student in School of Civil Engineering, Harbin Institute of Technology His interests are structural damage detection and structural health monitoring.

Xin Yan received his $\mathrm{PhD}$ in Civil Engineering from the Harbin Institute of Technology in 2010. He currently works as an Associate Research Fellow in China Highway Hi-tech Traffic Inspection and Certification Co., Ltd. His interest is structural health monitoring.

This paper is a revised and expanded version of a paper entitled 'Fractal theory based damage assessing method of acoustic emission test' presented at The 8th International Conference on Damage Assessment of Structure, Beijing, China, 3-5 August 2009.

\section{Introduction}

In the last decades, developing automated sensor-based structural health monitoring (SHM) systems for accurately detecting, locating and assessing damage has received much attention. The efforts to implement SHM systems have been rewarded by an increasing number of sensors developed. Compared with other sensors used in SHM such as fibre optical sensors, piezoelectric ceramic (PZT) sensors have revealed to be a useful sensor for SHM of civil structures which has the advantages of simplicity, low cost, quick response and high reliability.

Generally, there are two major types of piezoelectric-based SHM approaches: active monitoring and passive monitoring. The active monitoring approach needs actuators. It contains two major methods: electro-mechanical impedance method and wave propagation method. The impedance-based health monitoring approach was first proposed by Sun et al. (1995). In the method, a PZT patch can be used as both an actuator and a sensor based on its electro-mechanical coupling property. The method utilises the dependence of the electrical impedance of the PZT patch, on the mechanical properties of a structure. Wave propagation method studies the elastic wave generated by actuators embedded in or bonded on the structures, which propagates within the structure and received by the sensors. This method was first proposed by Chang of Stanford University (Roh and Chang, 1999; Wang and Chang, 1999). Song et al. (2007) embedded piezoceramic transducers in the concrete structure and utilised this method for damage detection of a long reinforced concrete bridge bent-cap.

For the piezoelectric-based for passive monitoring, PZT has the character of high impedance and it is feasible to measure the dynamic and mechanical variable of a structure. As passive monitoring method of practical interest, acoustic emission (AE) tests usually employ PZT material as mechanical and electrical transducers to monitor metallurgical transformation, dislocation movements, plastic yielding and micro-cracking 
of structures, etc. AE is a naturally occurring phenomenon of elastic waves generated when there is a rapid release of energy in a material or on its surface (Fang and Berkovits, 1995), for example as a result of plastic deformation and crack formation. AE monitoring is an effective non-destructive technique (Dhale and Khan, 2013) for detecting, locating and assessing damage on steel (Wang et al., 2015; Nemati et al., 2015), concrete (Nor et al., 2011; Pazdera et al., 2017) and FRP structures (Li et al., 2011; De Oliveira and Marques, 2008), etc.

In AE signal processing, signal measurement parameters are typically used, and the most widely used measurement parameters include counts, amplitude, duration, rise time and the measured area under the rectified signal envelope (alternatively counts energy). Recently, with the advance of signal processing and computer technology, waveformbased AE detection becomes practical. These introductions of these technologies were inspired by conventional signal processing methods, such as spectrum analysis, artificial intelligent methods (Prosser, 1995; Ali et al., 2016), wavelet transform (Ni and Iwamoto, 2002; Liu et al., 2015) and data fusion (Niri et al., 2012), which has the potential to detect and characterise damage more accurately. Poddar and Giurgiutiu (2017) investigated the detectability of the crack length from the recorded AE signal in plate structures based on the wave propagation physics.

It is found that the AE signals typically are fractals, and thereby the fractal theory has been explicitly introduced in AE signal processing in recent years. Silva et al. (2005) presented several fractal indices, which are related to the Hurst analysis, detrended fluctuation analysis, minimal cover analysis. The box-counting dimension analysis was employed to characterise the different failure modes. Paparo and Gregori (2004) demonstrated the correlation between the fractal dimensions (FD) and the order of the space distribution of the prime AE sources. Li et al. (2011) also investigated a FD-based damage index for damage quantification and failure warning of FRP cables.

The main contributions of this study are demonstrated as follows: firstly, the fractal analysis of signal is presented and the fractal theory-based damage assessing index is proposed through the fractal analysis. Then, the AE tests on pseudo-static experiment of three specimens are performed for validation, where several AE-PZT patches are bonded to the different places of the surface of the specimens to monitor the AE signals. It will be demonstrated that the proposed damage assessing index can assess damage evolution as well as the damage locations effectively. The differences of the damage evolution among the three types of specimens will also be discussed in the paper.

\section{Method}

\subsection{Fundamentals of the method}

To compute FD of a signal, the FD arithmetic proposed by Higuchi (1988) is adopted in this study, which is suitable for analysing an irregular time series. We first define the length of time series of an AE signal, $X$, as (Higuchi, 1988)

$$
L_{m}(k)=\left\{\left(\sum_{i=1}^{[(N-m) / k]}|X(m+i k)-X(m+(i-1) k)|\right) \cdot \frac{N-1}{[(N-m) / k] \cdot k}\right\} / k
$$


where $N$ refers to the number of sampling points; $k$ and $m$ are the interval time and initial time, respectively; [ ] denotes the Gauss notation. The average value over $k$ sets of $L_{m}(k)$ is given by:

$$
L(k)=\frac{1}{k} \sum_{m=1}^{k} L_{m}(k)
$$

This is because for any time interval $k, k$ sets of new time series can be generated, where each starts from the initial time $m(m=1,2, \ldots, k)$.

If the average value $L(k)$ has linear relationship with $k^{-F D}$, we consider the signal $X$ as fractal, that is

$$
L(k)=b \cdot k^{-F D}
$$

where $b$ is the curve length of the original signal:

$$
b=L(1)=\sum_{i=1}^{N-1}|X(i+1)-X(i)|
$$

The quantity $b$ is related with the fluctuation amplitude of a signal.

Then, $F D$ can be derived from equation (3), as

$$
F D=-[\ln (L(k))-\ln (b)] / \ln (k)
$$

It is indicated that $F D$ is the slope of the straight line. It is known any signal can be denoted by the following function

$$
X=\sum_{j=1}^{\infty} A_{j} \sin \omega_{j} t
$$

where $A_{j}$ is the amplitude at frequency $\omega_{j}$, then the curve length of the signal can be obtained by using equation (3) and the FD value can be computed from the following form:

$$
\begin{aligned}
& F D=-[\ln (L(k))-\ln (b)] / \ln (k) \\
& =\frac{\ln \sum_{j=1}^{\infty}\left[A_{j} \sum_{i=1}^{N-1}\left(\left|\sin \omega_{j} t_{i+1}-\sin \omega_{j} t_{i}\right|\right)\right]-\ln \sum_{j=1}^{\infty}\left\{\frac{A_{j}}{k} \sum_{m=1}^{k}\left[\sum_{i=1}^{[[(N-m) j k]}\left(\left|\sin \omega_{j} t_{m+i k}-\sin \omega_{j} t_{m+t i-1) k}\right|\right) \cdot \frac{N-1}{[(N-m) / k] \cdot k}\right] / k\right\}}{\ln k} \\
& =\frac{\ln \sum_{j=1}^{\infty}\left[n A_{j} \sum_{i=1}^{N-1}\left(\left|\sin \omega_{j} t_{i+1}-\sin \omega_{j} t_{i}\right|\right)\right]-\ln \sum_{j=1}^{\infty}\left\{\frac{n A_{j}}{k} \sum_{m=1}^{k}\left[\sum_{i=1}^{\left[L_{(N-m) / k}\right.}\left(\left|\sin \omega_{j} t_{m+i k}-\sin \omega_{j} t_{m+i(i-1) k}\right|\right) \cdot \frac{N-1}{[(N-m) / k] \cdot k}\right] / k\right\}}{\ln k} \\
& =\frac{\ln \left\{\sum_{j=1}^{\infty}\left[n A_{j} \sum_{i=1}^{N-1}\left(\left|\sin \omega_{j} t_{i+1}-\sin \omega_{j} t_{i}\right|\right)\right] / \sum_{j=1}^{\infty}\left[\frac{n A_{j}}{k} \sum_{m=1}^{k}\left(\sum_{i=1}^{[(N-m) \cdot k]}\left(\left|\sin \omega_{j} t_{m+k+k}-\sin \omega_{j} t_{m+t i-1) k}\right|\right) \cdot \frac{N-1}{[(N-m) / k] \cdot k}\right)\right] / k\right\}}{\ln k}
\end{aligned}
$$

where $n$ is a constant value. Equation (7) indicates that for two signals with the same frequency distribution and the relative amplitudes at each frequency component in the two signals are the same, the $F D$ values for the two will be identical. In other words, the $F D$ values are only dependent on the relative amplitudes of each frequency component contained in a signal. 
In equation (7), we set

$$
\begin{aligned}
& \lambda=\frac{A_{j} \sum_{i=1}^{N-1}\left(\left|\sin \omega_{j} t_{i+1}-\sin \omega_{j} t_{i}\right|\right)}{\frac{A_{j}}{k} \sum_{m=1}^{k}\left(\sum_{i=1}^{[(N-m) / k]}\left(\left|\sin \omega_{j} t_{m+i k}-\sin \omega_{j} t_{m+(i-1) k}\right|\right) \cdot \frac{N-1}{[(N-m) / k] \cdot k}\right) / k} \\
& =\frac{A_{j} \sum_{i=1}^{N-1}\left(\left|\sin \omega_{j} \cdot(i+1) \cdot \Delta t-\sin \omega_{j} \cdot i \cdot \Delta t\right|\right)}{\frac{A_{j}}{k} \sum_{m=1}^{k}\left(\sum_{i=1}^{[(N-m) / k]}\left(\left|\sin \left(\omega_{j} \cdot i \cdot(k \Delta t)+\omega_{j} \cdot t_{m}\right)-\sin \left(\omega_{j} \cdot(i-1) \cdot(k \Delta t)+\omega_{j} \cdot t_{m}\right)\right|\right) \cdot \frac{N-1}{[(N-m) / k] \cdot k}\right) / k}
\end{aligned}
$$

Figure 1 Time series of signals (a) $X_{1}$, (b) $X_{2}$, (c) $X_{3}$ and (d) $X_{4}$

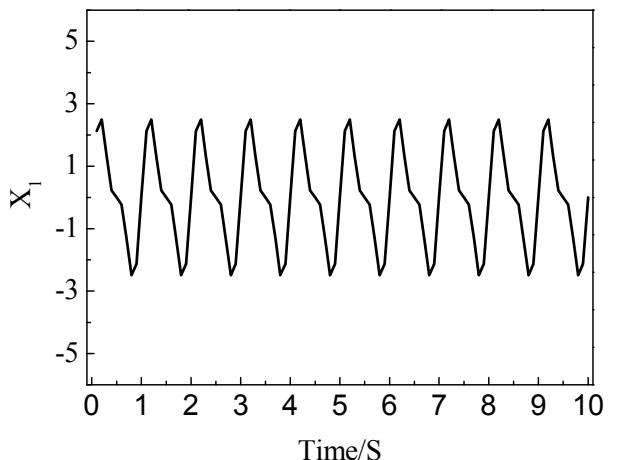

(a)

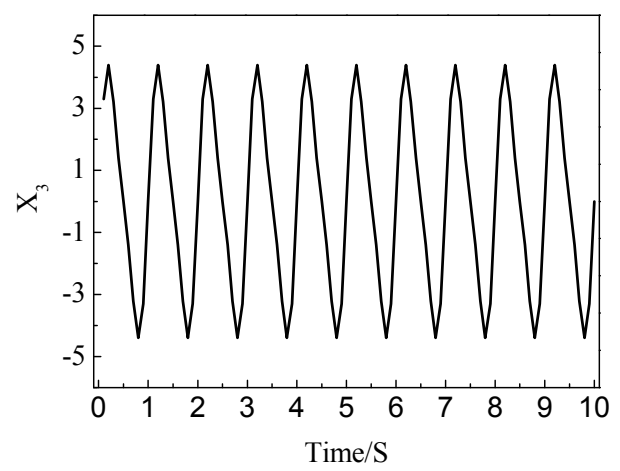

(c)

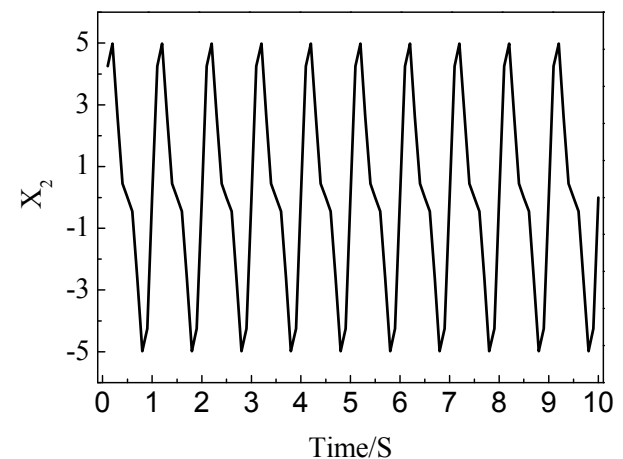

(b)

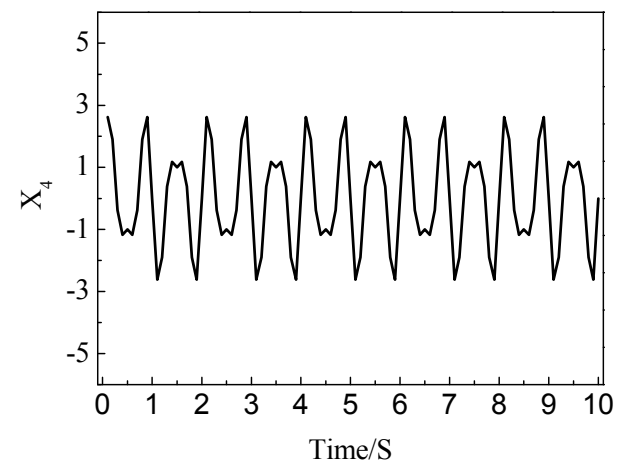

(d)

The property that the signal is fractal is valid over the time scales $0<k \Delta t \leq \frac{\pi}{\omega_{j}}$. It is seen that the bigger value of $\omega_{j}$, the larger value of $\lambda$ will be. As $F D=\ln \lambda$, higher frequencies also produce larger value of $F D$.

The characteristic that higher frequency components will induce larger $F D$ values of a signal will be demonstrated by using several harmonic signals. Assuming that signals $X_{1}$, $X_{2}, X_{3}$ and $X_{4}$ [see Figures 1(a), 1(b), 1(c) and 1(d)] are expressed as follows (see Figure 1): 


$$
\begin{array}{ll}
X_{1}=2 \sin 2 \pi t+\sin 4 \pi t & (0<t<10 s) \\
X_{2}=4 \sin 2 \pi t+2 \sin 4 \pi t & (0<t<10 s) \\
X_{3}=4 \sin 2 \pi t+\sin 4 \pi t & (0<t<10 s) \\
X_{4}=2 \sin 3 \pi t+\sin 5 \pi t & (0<t<10 s)
\end{array}
$$

Obviously, the first three signals contain two frequency components of $1 \mathrm{~Hz}$ and $2 \mathrm{~Hz}$, and the relative amplitude ratio between the high and low frequency components are $1 / 2$ for the first two signals, and $1 / 4$ for signal $X_{3}$. While for signal $X_{4}$, the two frequency components are $1.5 \mathrm{~Hz}$ and $2.5 \mathrm{~Hz}$, and the relative amplitude ratio between the high and low frequency components are $1 / 2$.

Figure 2 Fractal analysis results of the four signals in Figure 1

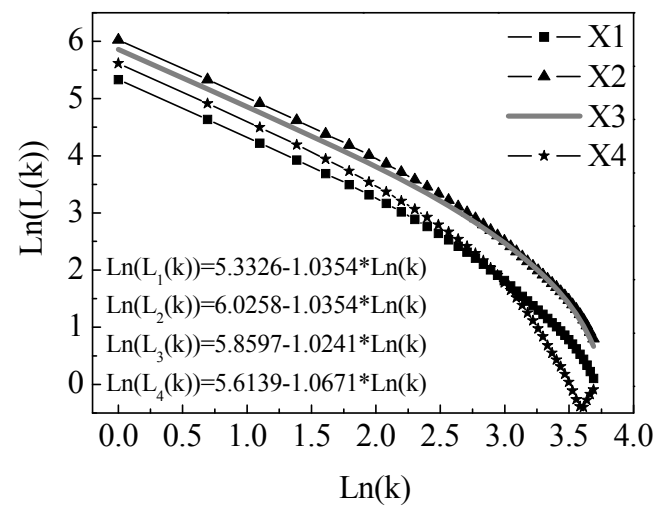

The sampling frequency for all the four signals is all $100 \mathrm{~Hz}$. From the fractal analysis in Figure 2, the four signals are fractal when the time intervals are from 1 to 10 . From equation (4), the $b$ values for the four signals are computed as 5.3326, 6.0258, 5.8597 and 5.6139, respectively; and the $F D$ values are 1.0354, 1.0354, 1.0241 and 1.0671 for the four signal by using equation (5). It is easily concluded that the $b$ values are related with the fluctuating intensity of a signal which is time domain information; while the $F D$ values are related with the frequency distribution of each signal which is frequency domain information. Therefore, the damage evolution of a structure can be quantified effectively by these two quantities of AE signal.

\section{$2.2 \quad$ FD-based damage assessment index}

As damage develops in a structure, the accumulated elastic energy in the structural material or on its surface will be released rapidly. For a time period, the time series of AE signal can be divided into several segments and a FD-based damage assessment index is defined as follows:

$$
J_{i}^{s}=\frac{\left(\sigma_{b}\right)_{i}}{(\bar{b})_{i}}+\frac{\left(\sigma_{F D}\right)_{i}}{(\overline{F D})_{i}}
$$

where $\sigma_{b}$ and $\bar{b}$ are the standard deviation and mean of the $b$ values for all signal segment, respectively; while $\sigma_{F D}$ and $\overline{F D}$ are the standard deviation and mean of the $F D$ 
values for all signal segment, respectively. For the whole damage evolution period of a structure, a cumulative damage assessment index can be further defined as

$$
J^{C}(j)=\sum_{i=1}^{j} J_{i}^{S}=\sum_{i=1}^{j} \frac{\left(\sigma_{b}\right)_{i}}{(\bar{b})_{i}}+\sum_{i=1}^{j} \frac{\left(\sigma_{F D}\right)_{i}}{(\overline{F D})_{i}}
$$

where $j=1,2, \ldots, N ; N$ is the total amount of time periods considered in the whole damage evolution period.

\section{Experimental validation}

\subsection{Experimental setup}

In order to verify the applicability and efficacy of the proposed FD-based damage index for AE signals, experiments on three specimens are conducted. Experimental set-up and loading regime are presented in the following.

\subsubsection{Specimens}

In the experiment, the test specimens are columns, including one reinforced concrete column (RCC), one reinforced nano-concrete tube (NMRCn4) and one glass fibre reinforced polymer (GFRP) tube filled with concrete (GFRPC).

The GFRP tube fabricated by filament-wound with e-glass fibres/epoxy resin in the hoop direction with 5 plies $(0.385 \mathrm{~mm}$ per ply), is $1,580 \mathrm{~mm}$ height and $150 \mathrm{~mm}$ inner diameter. The e-glass fibres used are RO99 2200 P122 manufactured by Beijing Saint-Gobain Vetrotex Glass Fiber Co. Ltd. The matrix is normally-used diphenol propane epoxy resin solidified by the diethylene triamine and the volume percentages of the fibres and epoxy are $45 \%$.

Figure 3 Sizes of test specimens and PZT detector localisations

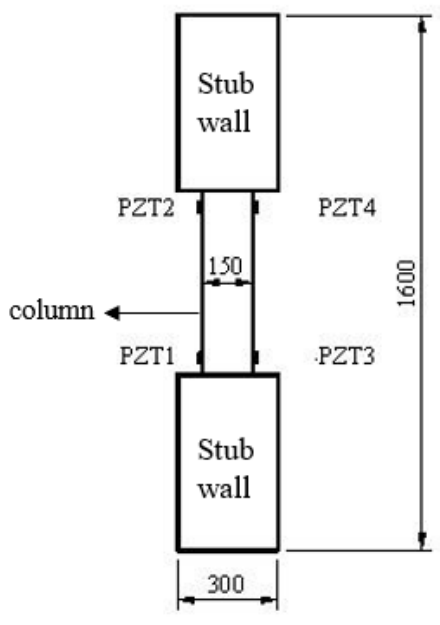

Side view

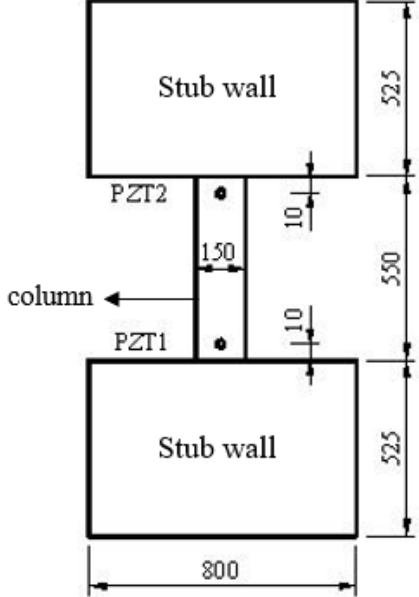

Front view 
The GFRP tubes are filled with concrete to form the test specimens. For loading, reinforced concrete beams at the upper and bottom ends of GFRP tubes are casted to embed the both ends of GFRP tubes into the RC beams. The column has a total height of $1,600 \mathrm{~mm}$ and a clear height of $550 \mathrm{~mm}$. The GFRP tubes are fully embedded into the reinforced concrete beams with only $10 \mathrm{~mm}$ left from the top and bottom surfaces of the reinforced concrete beams. The detailed information of the test specimens is illustrated in Figure 3, where the three specimens have the same sizes and sensor placements.

After the assembly of columns, concrete with 28-day cylinder strength of $63.7 \mathrm{MPa}$ was filled into the composite columns. For comparison, one RCC and one reinforced nano-concrete tube with the same size were fabricated at the same time. The column has four longitudinal bars with a diameter of $12 \mathrm{~mm}$, and hoop reinforcements at intervals of $60 \mathrm{~mm}$. The longitudinal ratio of reinforcement of the column is $2.56 \%$. The details of the test specimens are shown in Table 1.

Table 1 Outline of details of the test specimens

\begin{tabular}{lcccc}
\hline Specimen & Type & Number & Steel tube & GFRP tube \\
\hline CFFTn6 & concrete-filled GFRP & 1 & N/A & $1.925 \mathrm{~mm} / 5$ plies/ \\
& tube & & & $0.385 \mathrm{~mm}$ per ply \\
RCn4 & reinforced concrete & 1 & N/A & N/A \\
NMRCn4 & reinforced nano-concrete & 1 & N/A & N/A \\
Specimen & Winding mode & Compression & Shear & Confinement coefficient \\
& & ratio & span ratio & \\
CFFTn6 & [90]5 & 0.6 & 1.83 & 0.341 \\
RCn4 & N/A & 0.4 & 1.83 & N/A \\
NMRCn4 & N/A & 0.4 & 1.83 & N/A \\
\hline
\end{tabular}

\subsubsection{Test setup and loading regime}

The specimens are tested using the pseudo-static test facility. Firstly, they are applied a sustained axial load. In this study, two axial compression ratios are selected, which are 0.4 and 0.6 , as listed in Table 1. The axial loads are corresponding $450 \mathrm{kN}$ and $675 \mathrm{kN}$, respectively. Then, the specimens are applied reciprocating horizontal lateral loads with the loading method of force-displacement double-control, and the horizontal loads are controlled by force before the yielding of the specimen. Once the specimen reaches yielding, the horizontal loads are then switched to be controlled by displacement until failure of the specimen. The axial load is recorded by force sensor; the horizontal force is recorded by the actuator self and the displacement are collected by displacement transducer LVDT. The measurement system is shown in Figure 3.

The AE signals generated from the damage occurring and development of specimen are monitored by PZT patches. Four PZT patches are bonded on the surface of each column. Two are located at the upper end and front side of the column (the distance of the two PZT patches along the height of the column is $10 \mathrm{~cm}$ ). Another two are attached at the bottom end back side of the column (the distance between these two PZT patches is also $10 \mathrm{~cm}$ ), as shown in Figure 3. The sampling frequency for the AE monitoring is $20,000 \mathrm{~Hz}$. 


\subsection{Results and discussion}

\subsubsection{AE signal received by PZT patches}

In Figure 4, the time-history curves of AE signals of RCN4 specimen for early stage (horizontal load range is $0-\Delta_{y}, \Delta_{y}$ denotes the yielding displacement) and latter stage of damage evolution are shown. It is seen that AEs are released moderately and the amplitudes are small at early stage; but at latter stage the AEs are released acutely and the amplitudes are generally much larger.

Figure 4 Time-history curves of AE signals, (a) early stage of damage evolution (b) latter stage of damage development

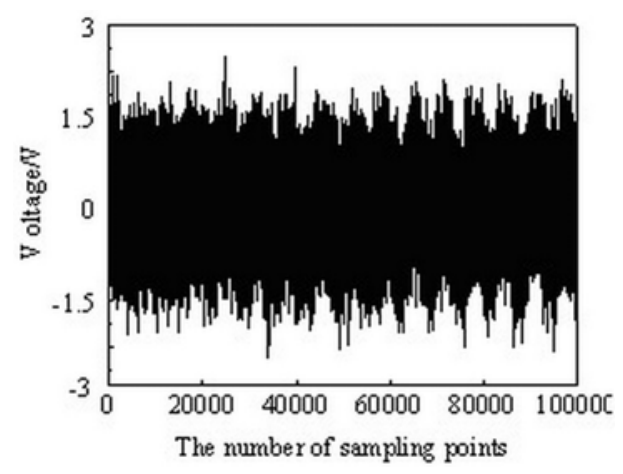

(a)

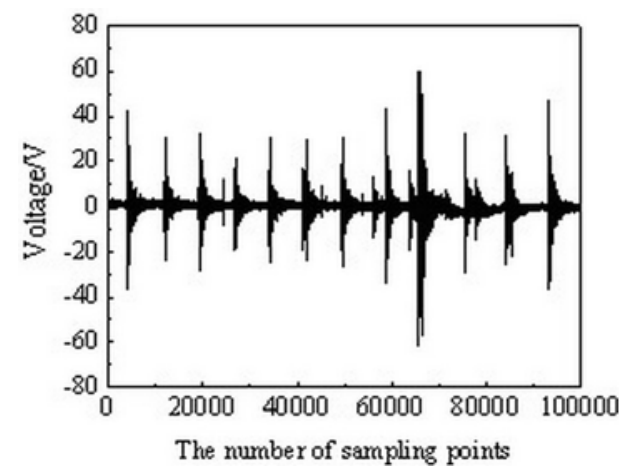

(b)

In Figure 5, the results of fast Fourier transform (FFT) of time-history signals in Figure 4 are presented. It is observed that the energy of the AE signal at latter stage is concentrated at low frequency range; while at early stage, the energy distribution for different frequency components is more like a uniform distribution. Therefore, the FD values at latter stage should be smaller than that at early stage. Note that there are three peaks appearing at the low frequency range of the early stage in Figure 5(a), this is presumably produced by the fibre fracture in the specimen.

Figure 5 FFT of AE signals in Figure 4, (a) early stage of damage evolution (b) latter stage of damage development

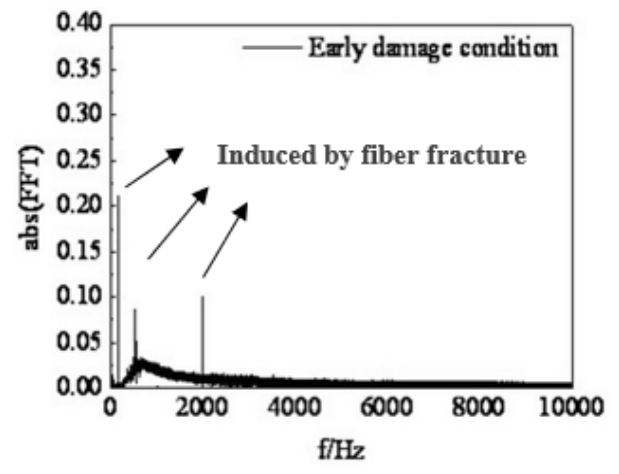

(a)

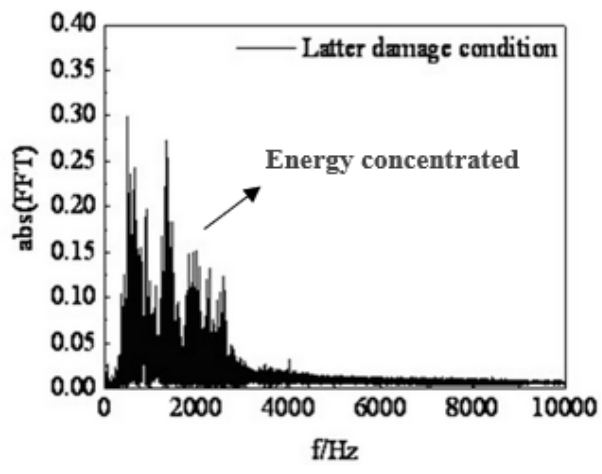

(b) 
Figure 6 The log curve length $\ln (L(k))$ versus log time interval $\ln (k)$ for seven equal-length time series at different damage evolution stages (a) very mild damage occurring, (b) mild damage development and (c) acute damage development

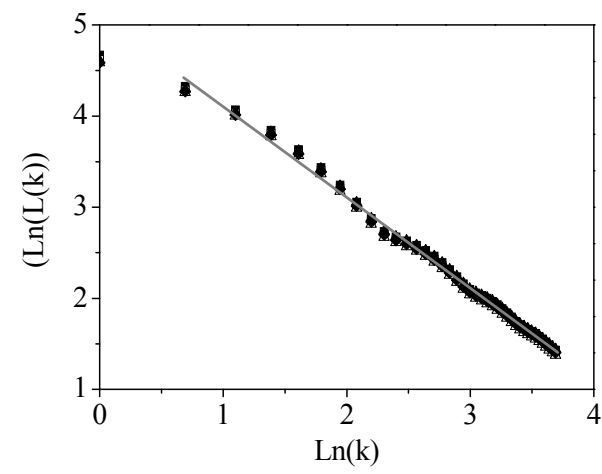

(a)

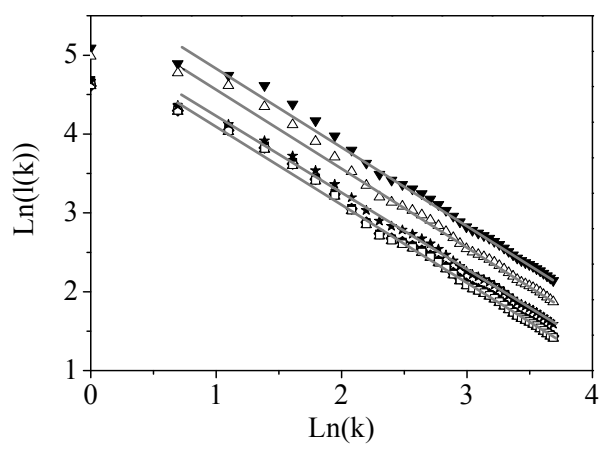

(b)

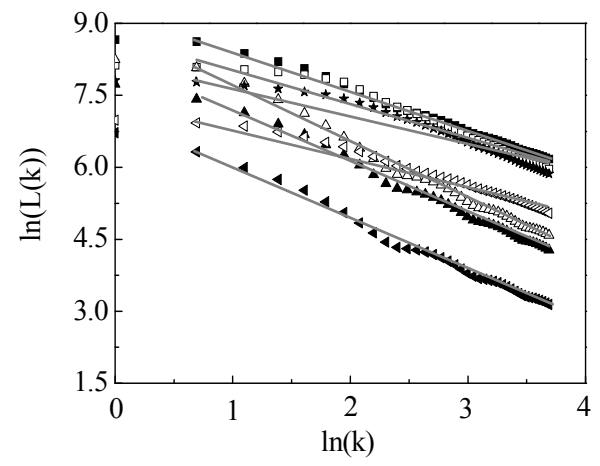

(c)

Notes: The markers denote the $\log L(k)$ values for various selected time intervals $k$ and each type of markers corresponds to a time series truncated. For each type of marker, the fitting line is provided to fit the markers of $\log L(k)$ versus $\log k$.

\subsubsection{FD values of AE signals}

In Figure 6, three different damage evolution stages of $\mathrm{RCn} 4$ specimen are analysed using fractal theory in equation (1)-(5). The AE time series of equal length of 5 seconds are truncated for $F D$ calculation. The time interval values $k$ are set from 1 to 40 , and the corresponding $L(k)$ values are computed for each $k$. In each figure, several fitting lines are also provided to fit the logarithm values of $L(k)$ versus $\log k$. As strictly strait lines are obtained, the AE signals investigated here are fractals.

It is demonstrated that at the stage with very mild damage occurring $\left(\Delta_{\max } / \Delta y<1\right)$, the straight lines of $\ln (L(k))$ versus $\ln (k)$ for different time series overlap with each other [Figure 6(a)]. This is due to the fact that only mild AEs occur at this stage. As damage continue to develop but still be slight $\left(\Delta_{\max } / \Delta y=1\right)$, the approximate lines of $\ln (L(k))$ versus $\ln (k)$ for different time series do not overlap but still be parallel [Figure 6(b)], implying that the $F D$ values are still the same but the $b$ values are district. Note that the two stages above correspond with the early stage of damage development in Figures 5 
and 6. When damage develops acutely $\left(\Delta_{\max } / \Delta y=3\right)$, it is seen that the curves of $\log (L(k))$ versus $\log (k)$ for different time series are reticular [see Figure 6(c)], because both the $F D$ and $b$ values are difference for various time series. Note that the first two stages and the last stage above correspond with the early and latter stage of damage development in Figures 5 and 6.

Figure 7 The average values of $F D$ and $b$ for the whole experiment process for specimens, (a) RCn4, (b) NMRCn4 and (c) CFFT
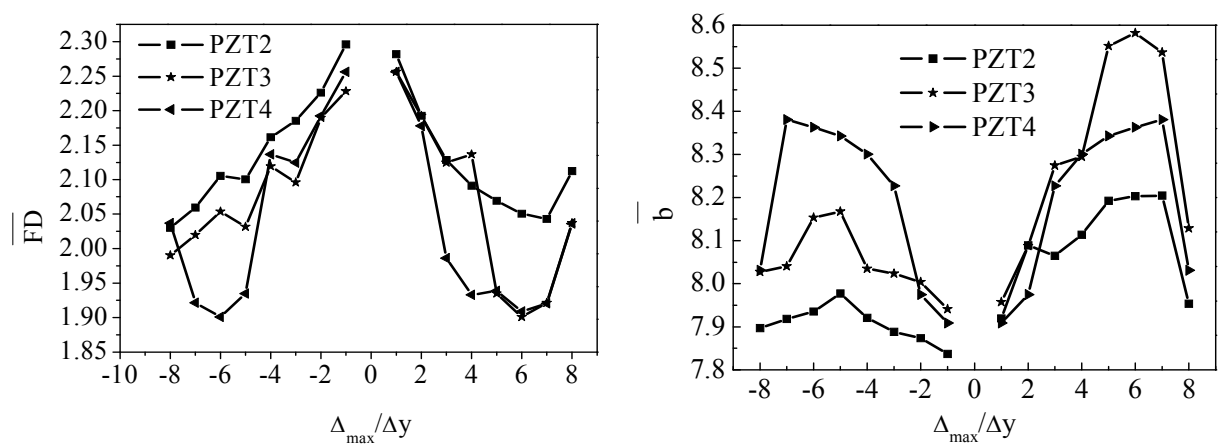

(a)
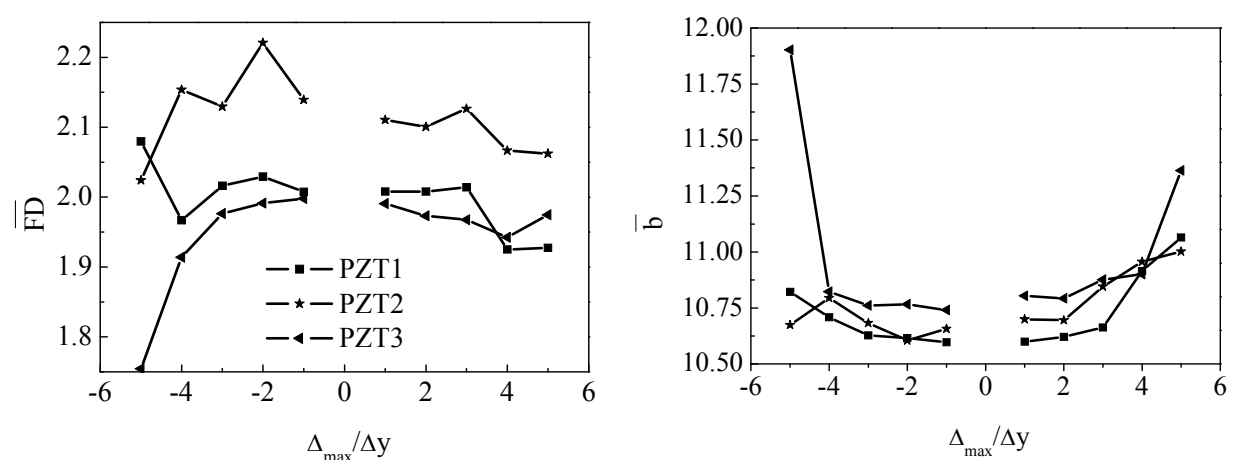

(b)
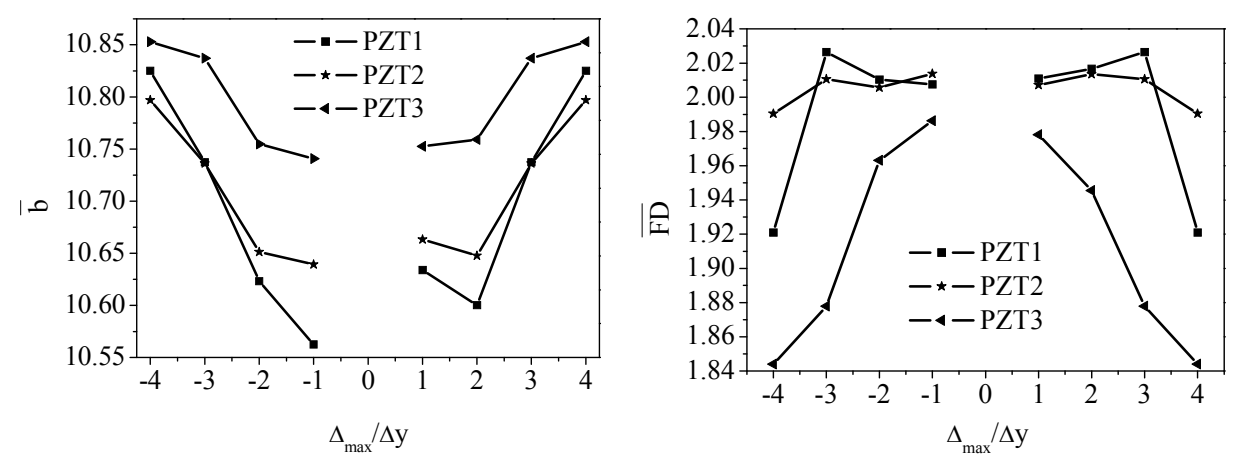

(c)

Note: $\Delta_{\max }$ is the maximal displacement for the loading process; $\Delta y$ is the yielding displacement. 
Figure 8 The profile of FD-base AE assessing index for a whole loading process of different specimens, (a) RCn4 (b) NMRCn4 (c) CFFT

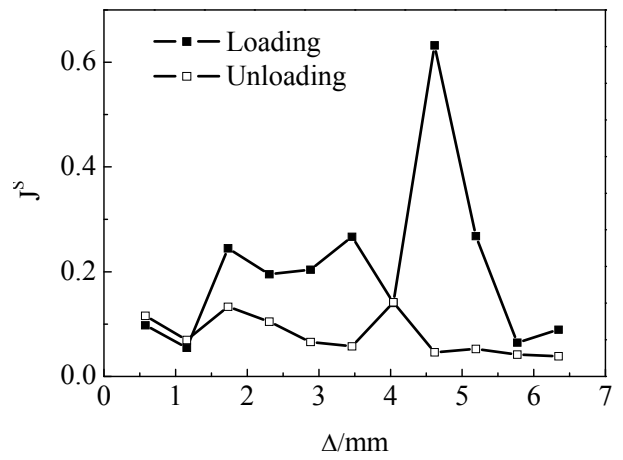

(a)

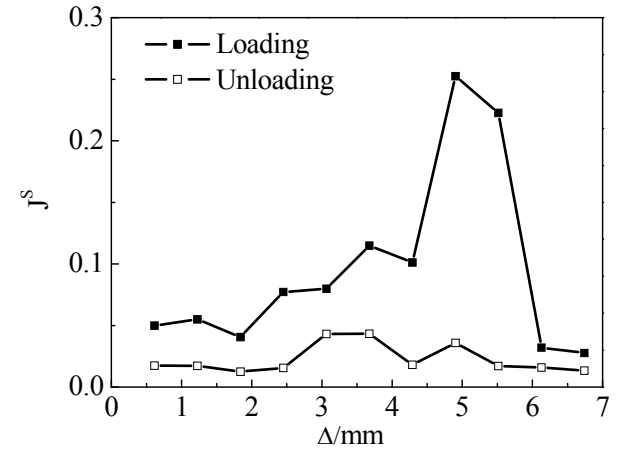

(b)

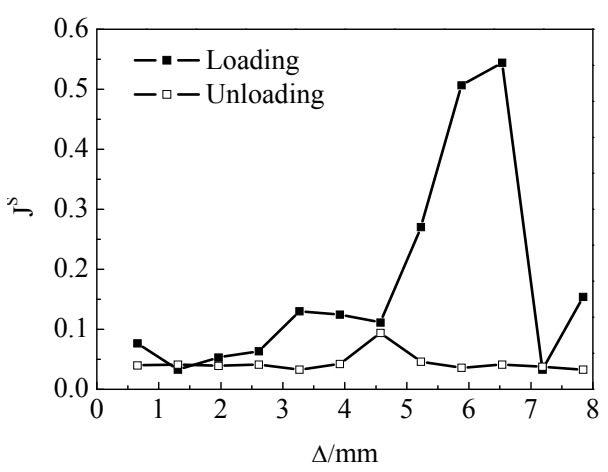

(c)

The average values of $F D$ and $b$ of the time series measured by PZT2, PZT 3 and PZT4 during the loading process are also shown in Figure 7. It is seen that the mean values of $F D$ have decreasing but $b$ have increasing trends for all specimens, indicating as damage develops the AE frequency distribution becomes lower but the fluctuation intensity goes up. This is consistent with the results in Subsection 3.2.1.

\subsubsection{Damage assessment of the whole loading and unloading process}

In Figure 8, the damage assessing index curves for a whole loading and unloading process (the first cycle and $\Delta_{\max } / \Delta y=2$ ) are shown. It is demonstrated that the whole loading and unloading process can be distinguished into four periods by observing the changing trend. The first period is the first half part of loading process. At this period, the values of $J^{S}$ are small because there are little cracks taking place for the origination of AEs. The second stage is the latter half part of the loading process. The values of $J^{S}$ are very large which indicates that a large amount of AEs are generated and released rapidly, corresponding to intense damage development. The third period is the first half part of unloading process where the values of $J^{S}$ are very small. The fourth is the second half unloading process, and the values of $J^{S}$ are generally larger than those of the third period because of the closing and friction of the fracture surface. In a word, the value changes of index $J^{S}$ are enable to characterise the damage occurrence and evolution of the structure. 
Figure 9 Comparison between the $P-\Delta$ curve and $J^{S}-\Delta$ curve for different specimens, (a) RCn4 (b) NMRCn4 (c) CFFT

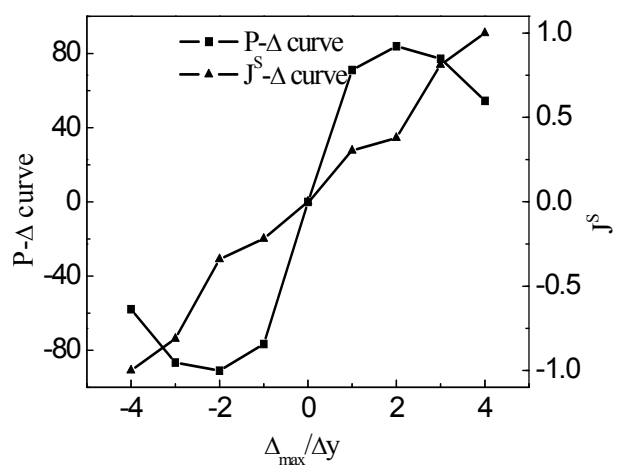

(a)

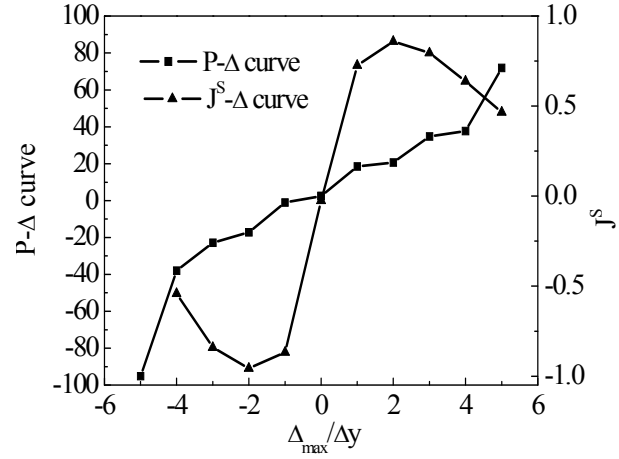

(b)

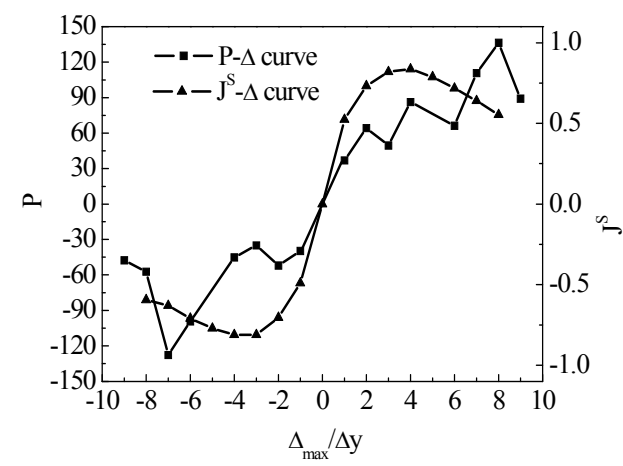

(c)

\subsubsection{Comparison of the proposed damage assessment indices with other damage indices}

\subsubsection{Comparison between the $P-\Delta$ curve and damage assessing index curve}

The load-displacement $P-\Delta$ curve and $J^{S}-\Delta$ curve of the loading process are compared in Figure 9. It is seen that an abrupt increase of the damage assessing index $J^{S}$ coincide with the descending segment of the $P-\Delta$ curve. The reason behind this is that both the descending segment of the $P-\Delta$ curve and the abrupt increase of warning index denote an obvious loss of stiffness in the structure. Therefore, the damage assessing index $J^{S}$ has the ability to warn structure failure. It is also seen for index $J^{S}$, different time are needed to reach the warning location (abrupt increase of $J^{S}$ ) for the three specimens. The warning time for CFFT is the longest, denoting the CFFT has the best ductility among the three.

\subsubsection{Comparison between Park-Ang damage index and cumulative damage assessing index}

The Park-Ang damage index (Park and Ang, 1985), which is widely used in concrete structures under earthquake ground motions, is defined as: 


$$
D=\frac{\delta_{M}}{\delta_{u}}+\frac{\beta}{Q_{y} \delta_{u}} \int d E
$$

where $\delta_{u}$ denotes the ultimate deformation under monotonic loading; $Q_{y}$ is the calculated yield strength; $d E$ is the incremental absorbed hysteretic energy; $\delta_{M}$ refers to the maximum deformation and $\beta$ is a non-negative parameter.

Figure 10 Results of Park-Ang damage index $D$ and index $J^{C}$ of PZT sensors for different specimens, (a) RCn4 (b) NMRCn4 (c) CFFT

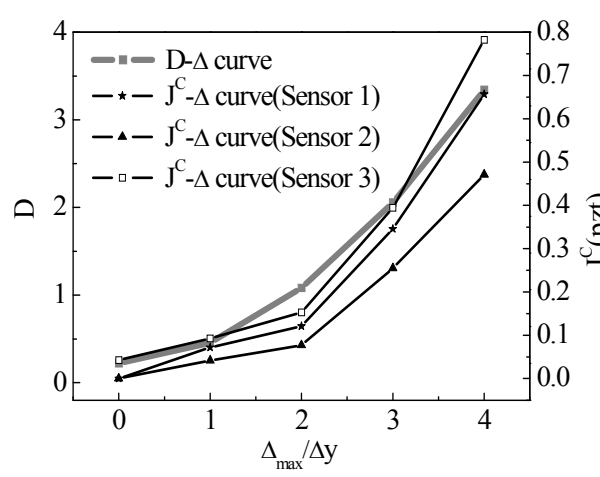

(a)

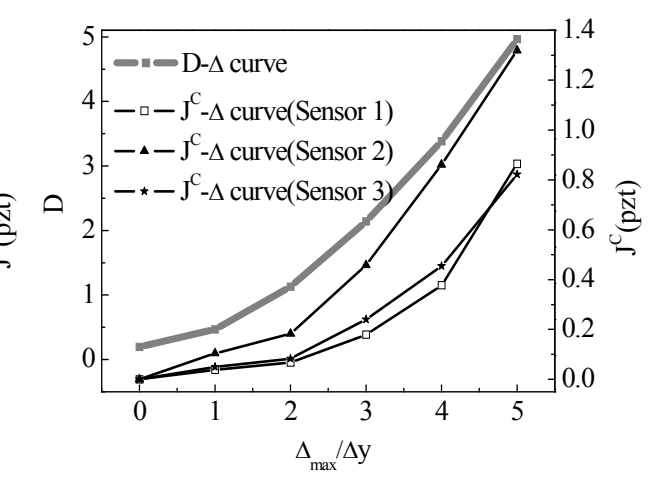

(b)

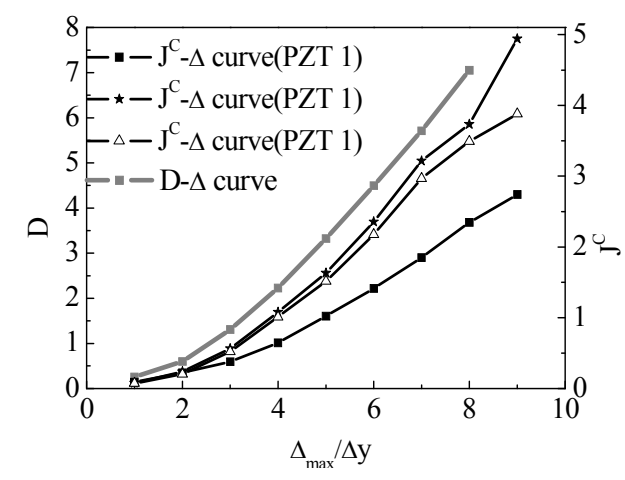

(c)

In Figure 10, the values of damage index $D$ and $J^{C}$ for each loading process (the displacement is positive) are shown. It is easily found as $\Delta_{\max } / \Delta y=3$ for RCn 4 specimen and $\Delta_{\max } / \Delta y=4$ for specimen NMRCn4 specimen, the Park-Ang damage index $D \geq 1$, which signifies the total failure of a structure. What is interesting is that at this time the index $J^{C}$ show an abrupt increase. Therefore, the two damage indexes behave a similar change trend for each specimen and the proposed damage assessing index $J^{C}$ is also an effective index to quantify accumulative damage development as the Park-Ang damage index. 


\subsubsection{Damage localisation}

For damage localisation purpose, a pencil lead broken on an FRP blade was performed to study the influence of the propagate distance between the damage occurring locations and sensors on damage assessing index $J$. A pencil lead of approximately $3 \mathrm{~mm}$ long and $2 \mathrm{~B}$ hardness was broken at various locations along the length direction of the blade, then the damage assessing indices $J$ of a fixed sensor can be obtained as shown in Figure 11.

Figure 11 The influence of the distance between the damage occurring place and sensor locations on (a) the index value $J$ and (b) the two factors of index $J$

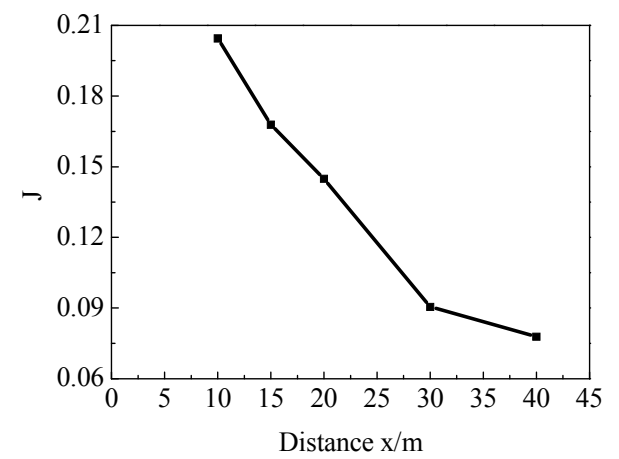

(a)

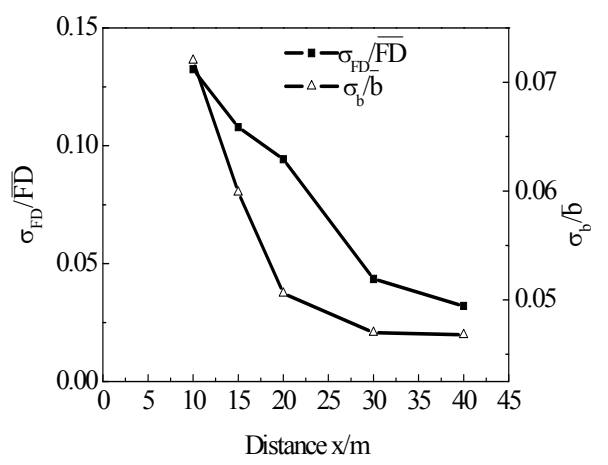

(b)

It is seen that as the pencil lead broken places become farther from the received sensor, the values of damage assessing index $J$ become smaller, because both the two factors $\sigma_{F D} / \overline{F D}$ and $\sigma_{b} / \bar{b}$ decrease. This is because the AE wave energy is diffused, diffracted and dissipated as the wave spread around. The abrupt frequency components of the AE signal become smoother, which results in the smaller values the standard deviations of $F D$ and $b$. By utilising the effect of the signal travel distance on the damage index, it is feasible to employ damage assessing index $J$ to localise damage by AE signal. Note that the largest distance between the damage and sensors is $40 \mathrm{~m}$ in Figure 11, which is much larger than the specimen size in Figure 3, thereby the conclusion drawn from Figure 11 can be utilised in damage localisation for the specimens in Figure 3.

For validation of the effectiveness of damage localisation, a loading process of NMRCn 4 specimen was performed. The specimen had severe damage close to sensor 2 , which had a small piece of concrete falling off from the specimen as shown in Figure 12(a). In Figure 12(b), the values of the damage assessing index of sensors 1-4 are demonstrated. It is found sensor 2 and sensor 3 have the largest and smallest values, which is consistent with our prediction, because their locations are closest and farthest from the falling piece of concrete, respectively. Note that, in the test, it is feasible for us to judge the distances of the possible damage from various sensors approximately by employing the proposed damage assessing index, which is not the exact damage location information. 
Figure 12 Damage assessing index $J$ at a given loading process of the specimen of NMRCn4 for different AE-PZT sensors (see online version for colours)

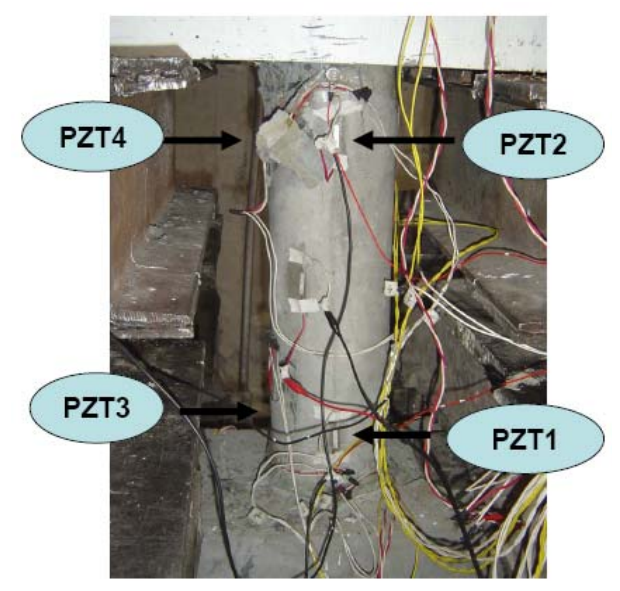

(a)

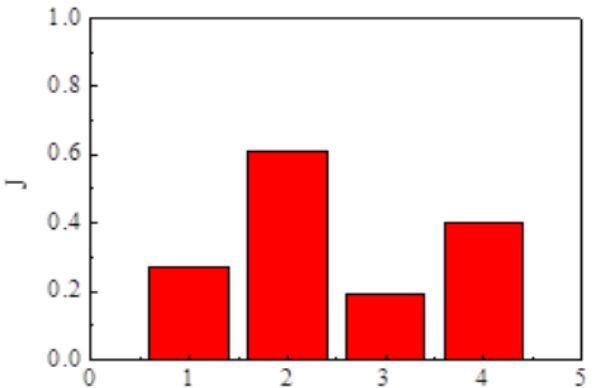

(b)

\section{Conclusions}

$\mathrm{AE}$ is an effective SHM technique for detecting, locating and characterising structural damage. In this paper, it was found that not only FD, but also the signal lengths are related with the damage evolution, because they present the frequency and tine domain information, respectively. Based on the analysis, a FD-based damage assessing index was proposed.

The AE tests on pseudo-static experiments of a RCC, a reinforced nano-concrete column and a concrete-filled GFRP tubes were performed for validation. For each specimen, there were several PZT patches bonded on different positions of the specimen surface to monitor AE signals of the structure. The AE signal analysis results showed that the proposed damage assessing index can assess damage evolution effectively. From the comparison studies, it is found the changing trend of the proposed damage assessing index are consistent with those of the load-displacement curve and Park-Ang damage index, and thereby it has the ability to warn structure failure. The distances of damage away from the sensors have been compared from the diversity of the values of the proposed damage assessing index since AE signals weaken more as the spread paths become longer. Overall, the experimental results demonstrate that the fractal theory-based damage assessing method is promising to be applied for practical $\mathrm{AE}$ monitoring and signal processing. 


\section{References}

Ali, Y., Ali, S.M., Rahman, R.A. and Hamzah, R.I. (2016) 'Acoustic emission and artificial intelligent methods in condition monitoring of rotating machine', The National Conference for Postgraduate Research 2016, University Malaysia Pahang.

De Oliveira, R. and Marques, A.T. (2008) 'Health monitoring of FRP using acoustic emission and artificial neural networks', Computers \& Structures, February, Vol. 86, Nos. 3-5, pp.367-373.

Dhale, A. and Khan, F. (2013) 'Application of acoustic emission technique in various field', International Journal of Engineering Research and Development, Vol. 7, No. 10, pp.80-85.

Fang, D. and Berkovits, A. (1995) 'Fatigue design model based on damage mechanisms revealed by acoustic emission measurements', Journal of Engineering Materials and Technology, Transactions ASME, Vol. 117, No. 2, pp.201-208.

Higuchi, T. (1988) 'Approach to an irregular time series on the basis of the fractal theory', Physica D: Nonlinear Phenomena, Vol. 31, No. 2, pp.277-283.

Li, H., Huang, Y., Chen, W.L., Ma, M.L., Tao, D.W. and Ou, J.P. (2011) 'Estimation and Warning of fatigue damage of FRP stay cables based on acoustic emission techniques and fractal theory', Computer-Aided And Infrastructure Engineering, Vol. 26, No. 7, pp.500-512.

Liu, X., Liang, Z., Zhang, Y.B., Wu, X.Z. and Liao, Z.Y. (2015) 'Acoustic emission signal recognition of different rocks using wavelet transform and artificial neural network', Shock and Vibration, Vol. 2015, No. 6, pp.1-14.

Nemati, N., Metrovich, B. and Nanni, A. (2015) 'Acoustic emission assessment of through-thickness fatigue crack growth in steel members', Adv. Struct. Eng., Vol. 18, No. 2, pp.269-283.

Ni, Q.Q. and Iwamoto, M. (2002) 'Wavelet transform of acoustic emission signals in failure of model composites', Engineering Fracture Mechanics, April, Vol. 69, No. 6, pp.717-728.

Niri, E.D., Farhidzadeh, A. and Solamone, S. (2012) 'Adaptive multisensor data fusion for acoustic emission source localization in noisy environment', Structural Health Monitoring, Vol. 12, No. 1, pp.59-77.

Nor, N.M., Bunnori, N.M., Ibrahim, A., Shahidan, S. and Soffian, N.S. (2011) 'Relationship between acoustic emission signal strength and damage evaluation of reinforced concrete structure', 2011 IEEE Symposium on Industrial Electronics and Applications, pp.308-313.

Paparo, G. and Gregori, G.P. (2004) 'Multifrequency acoustic emission (AE) for monitoring the time evolution of microprocesses within solids', in Thompson, D.O. and Chimenti, D.E. (Eds.): Proceeding on Reviews of Quantitative Nondestructive Evaluation, AIP, Melville, New York, Vol. 22, pp.1423-30.

Park, Y.J. and Ang, H.S. (1985) 'Mechanistic seismic damage model for reinforced concrete', Journal of Structure Engineering, Vol. 111, No. 4, pp.722-739, ASCE.

Pazdera, L., Topolar, L. and Mikulasek, K. (2017) 'Application of acoustic emission method during cycling loading of concrete beam', 9th International Workshop NDT in Progress, Prague, Czech Republic.

Poddar, B. and Giurgiutiu, V. (2017) 'Detectability of crack lengths from acoustic emissions using physics of wave propagation in plate structures', Journal of Nondestructive Evaluation, pp.36-41.

Prosser, W.H. (1995) 'Advanced waveform-based acoustic emission detection of matrix tracking in composites', Material Evaluation, Vol. 53, No. 9, pp.1052-1058.

Roh, Y.S. and Chang, F.K. (1999) Built in Diagnostics for Identifying an Anomaly in Plates Using Wave Scatting, Dissertation, Stanford University, USA.

Silva, F.E., Goncalves, L.L., Fereira, D.B.B. and Rebello, J.M.A. (2005) 'Characterization of failure mechanism in composite materials through fractal analysis of acoustic emission signals', Chaos, Solitons and Fractals, Vol. 26, pp.481-494. 
Song, G., Gu, H., Mo, Y.L., Hsu, T.T.C. and Dhonde, H. (2007) 'Concrete structural health monitoring using embedded piezoceramic transducers', Smart Mater. Struct., Vol. 16, pp.959-968.

Sun, F.P., Chaudhry, Z., Rogers, C.A. and Majmundar, M. (1995) 'Automated real-time structure health monitoring via signature pattern recognition', Proc. SPIE, Vol. 2443, pp.236-47.

Wang, C.S. and Chang, F.K. (1999) 'Built-in diagnostics for impact damage identification of composite structures', Proceedings of the 3rd International Workshop on Structural Health Monitoring, Stanford, USA, pp.612-621.

Wang, H.W., Yu, H.M., Xiao, H.Q., Han, Z.Y. and Luo, H.Y. (2015) 'Steel damage based on acoustic emission', Materials Research Innovations, Vol. 19, pp.288-291. 